\title{
Patient satisfaction with anesthesia for curative neoplastic surgery: the impact on quality of recovery Euroanaesthesia 2017
}

Carvalho B., Marinho R., Sousa M., Azevedo M., Santos A., Abelha F.

\section{Background and Goal of Study}

Patient reported outcomes have become one of the most important measures for assessing medical and surgical treatments. Patient satisfaction is an important tool for promoting improvements in clinical care. We aimed to evaluate if the the quality of recovery has influence on patients satisfaction after anaesthesia.

\section{Materials and Methods}

Observational prospective study

Inclusion criteria: adult patients undergoing curative neoplastic surgery.

Exclusion criteria: inability to give informed consent

Statistical analysis: The MannWhiney $U$ test, Chisquare or Fisher's exact test were used for comparisons.

\section{Avaliation of satisfaction:}

Postoperative Quality of Recovery Scale (PQRS) at D3

\section{Avaliation of quality of recovery:}

Postoperative Quality of Recovery Scale (PQRS): Pre-op, TI5, T40, DI and D3

Quality of Recovery I 5(QoR- I5): Pre-op and D I

\section{Results and Discussion}

From I33, 85\% of the patients were completely satisfied with anaesthesia care

\begin{tabular}{|l|c|c|c|} 
& $\begin{array}{c}\text { Complete } \\
\text { Satisfaction } \\
\mathbf{N}=113\end{array}$ & $\begin{array}{c}\text { Incomplete } \\
\text { Satisfaction } \\
\mathbf{N = 2 0}\end{array}$ & $\begin{array}{c}\text { Value } \\
\mathbf{p}\end{array}$ \\
\hline Age, median [P25-P75] & $\begin{array}{c}63,0 \\
{[53,5-72,5]}\end{array}$ & $\begin{array}{c}70,5 \\
{[57,5-78,8]}\end{array}$ & 0,156 \\
\hline Gender, $\mathbf{n}(\%)$ & $68(60,2)$ & $9(45,0)$ & 0,205 \\
\hline High risk surgery, $\mathbf{n}(\%)$ & $43(38,1)$ & $8(40,0)$ & 0,869 \\
\hline Ischemic heart disease, $\mathbf{n}(\%)$ & $14(12,4)$ & $3(15,0)$ & 0,490 \\
\hline Congestive heart, $\mathbf{n}(\%)$ & $10(8,8)$ & $3(15,0)$ & 0,306 \\
\hline Insulin dependent diabete, $\mathbf{n}(\%)$ & $6(5,3)$ & $1(5,0)$ & 0,717 \\
\hline Creatinin >2.0 $\mathbf{m g} / \mathbf{d L}, \mathbf{n}(\%)$ & $4(3,5)$ & $2(10)$ & 0,222 \\
\hline Cerebrovascular disease, $\mathbf{n}(\%)$ & $1(0,9)$ & $1(5,0)$ & 0,279 \\
\hline
\end{tabular}

Table I - Variable analysis for satisfaction
Results and Discussion

\begin{tabular}{|c|c|c|c|}
\hline Type of Anaesthesia & $\begin{array}{c}\text { Complete } \\
\text { Satisfaction } \\
\mathbf{N}=113\end{array}$ & $\begin{array}{c}\text { Incomplete } \\
\text { Satisfaction } \\
\mathbf{N = 2 0}\end{array}$ & $\begin{array}{c}\text { Value } \\
\mathbf{p}\end{array}$ \\
\hline General Anaesthesia, $\mathbf{n}(\%)$ & $82(72,5)$ & $9(45,0)$ & $\mathbf{0 , 0 1 4}$ \\
\hline Regional Anaesthesia,n(\% & $5(4,4)$ & $5(25,0)$ & $\mathbf{0 , 0 1}$ \\
\hline
\end{tabular}

Table 2 - Type of anaesthesia and Satisfaction

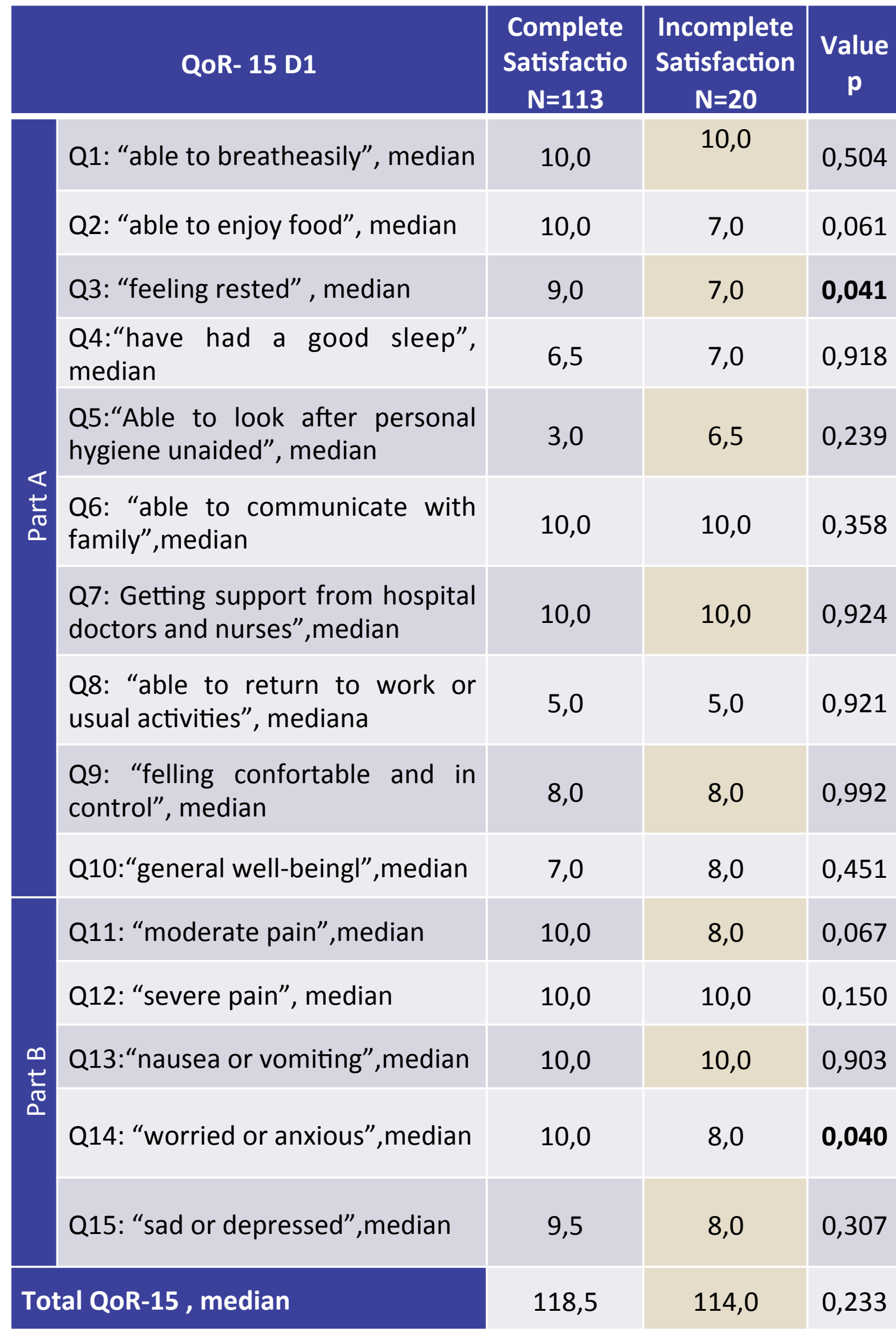

Table 3 - QoR-I5 variable analysis

\section{Conclusion}

Patients with incomplete satisfaction had a poor quality of recovery in two items of the QORI5 score at DI.Type of anaesthesia played an important role in neoplastic patient's satisfaction as well as feeling less rested and more anxious. 